\title{
Adherence to a Treatment of Cranial Deformities with a Sensorised Brace Device
}

\author{
Antoni Oller, Juan López, Jesús Alcober \\ BarcelonaTech. \\ C/ Esteve Terrades, 7 - 0860 Castelldefels, Barcelona (Spain) \\ E-mail: antoni.oller@upc.edu, juan.lopez-rubio@upc.edu, jesus.alcober@upc.edu
}

\author{
Guillem Tarín \\ Alteraid, S.L \\ C/ Esteve Terrades, Castelldefels - Barcelona, Catalunya (Spain) \\ E-mail: guillem.tarin@alteraid.com
}

Lucero Rosas, Julio García

Fix You

Mexico D.F

E-mail: rocalucero@gmail.com, rockagv.juliocesar@gmail.com

Received: December 15, 2015

DOI: 10.5296/npa.v8i1.8732
Accepted: March 14, 2016 Published: March 31, 2016

URL: http://dx.doi.org/10.5296/npa.v8i1.8732

\begin{abstract}
Positional plagiocephaly, braquicephaly and scaphocephaly are cranial deformations that occur on infants during 4 to 18 months, heads of babies with these deformations are asymmetric and in severe cases they might develop problems with sight and hearing senses as well as chewing and learning skills. The treatment for these deformations in average and severe cases is the use of a custom made helmet that allows the cranium to recover its symmetry; the amount of correction made depends on the total daily hours of usage, becoming a key metric. Currently to know how many hours the helmet is used, parents are
\end{abstract}


asked every month the total number of hours in average, but this is not an accurate way to register this time of usage. Knowing this value would allow giving a better follow up for each patient and could also estimate the duration of treatment for each case. This work describes an experience based on biomedical and telematics engineering discipline combination, which allows defining a treatment procedure for children between 4 and 18 months old to the diagnosis, helmet design, manufacturing and tracking in a way of registering the adherence during the whole treatment.

Keywords: Deformational plagiocephaly, helmet, empowerment, cloud, adherence, compliance.

\section{Introduction}

Alteraid, S.L is an innovative spin-off born in the Polytechnic University of Catalonia (UPC - BarcelonaTech) that provides ICT solutions for managing the citizens' quality of life, using patient-centric approach tools to enable patient empowerment and allow improvements in patient engagement. FIX YOU is a start-up born in the National Polytechnic Institute of the city of Mexico that offers an integral solution to cranial deformities for children between 4 and 18 months old using an orthopaedic helmet with textile sensors that registers the utilization adherence and shares that information in a cloud with the corresponding stakeholders (relatives, medical staff, etc.) in the treatment through a mobile application.

The shape of the heads of those babies with plagiocephaly or brachycephaly [1], a type of cranial deformity, is not symmetric. It can provoke problems with the sense of sight and hearing, problems with chewing and also some troubles with learning. Additionally, they have got psychological effects, when being faced with a different aspect from other children. The way to correct these deformities is through physiotherapy and, in severe cases, the use of an orthopaedic helmet or cranial orthosis [2].

The use of the pediatric orthopaedic helmet, as it can be seen in Fig. 1, is prescribed to babies between 4 and 12 months old who present cranial flattening symptoms. It is used to recover the head symmetry and, as a consequence, avoid the problems described before. To obtain favourable results, with the minimum time, it is necessary to wear the orthopaedic helmet 20 hours per day during 6 months in average, and, for this reason, it is important to have available an adherence control system, which informs us with the maximum reliability and minimum hassle for the baby when using the helmet [3]. 


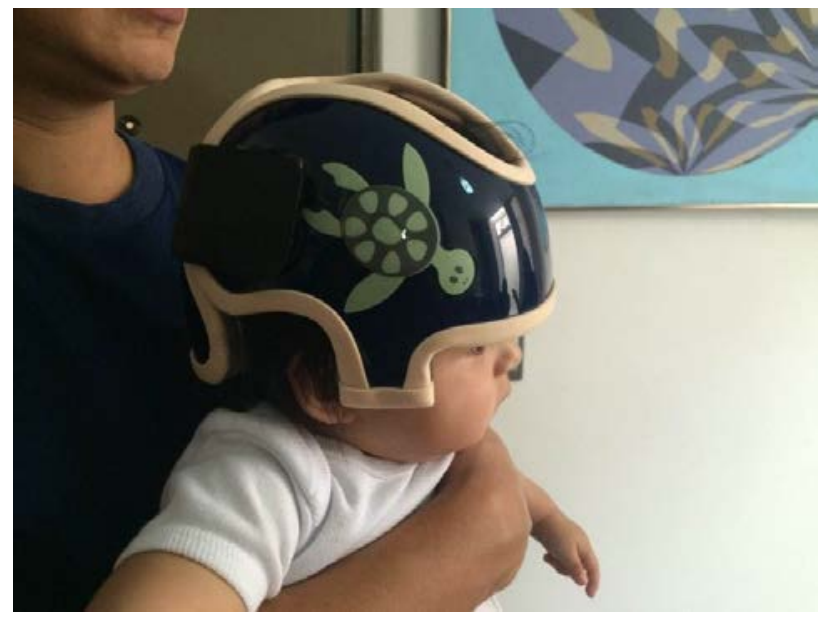

Figure 1. The pediatric orthopaedic helmet

This project was started in 2011 with a collaboration with the National Pediatric Institute. This public hospital is the most important in Mexico, which serves patients, coming from the whole country, with the characteristics described before. The project has been supervised by two pediatric neurosurgeons and the support of the hospital facilities [4].

This work describes the proposed adherence system to be integrated as part of the orthopaedic treatment for cranial deformations. The first stage implies sensor coupling and micro controller programming. Sensor coupling; select and design the circuit needed to change the voltage provided by the sensor into digital signal for further use in the micro controller. The micro controller used in the present work is an Arduino One, which is used to design the algorithm needed to register the time of usage and then transmit the data.

The test made in this stage is signal triggering from sensor to micro controller and time quantified by the micro controller.

In the second section the state of the art of the adherence systems will be described. In the third section, the challenges and objectives of the project are going to be listed. Then, the fourth section makes reference to the complete system architecture, and finally the results and conclusions of the current work will be analysed.

\section{State of the art of the adherence systems to treatments}

Cranial Orthosis affects some children during the first period of their lives. During the first six weeks, new born children might present an asymmetric head shape, most of them because of the baby passage through the birth canal, but it should be recovered passed this time, if it does not, a specialized physician needs to confirm a positional skull or cranial deformity.

The most common cranial deformity is plagiocephaly, which is shown as a flattening on one side of the back of the head and a flattening on the opposite side on the forehead. 
In order to diagnose the level of asymmetry (slight, mid or severe) there are two main ratios; the oblique cranial length ratio, which is used to determine the plagiocephaly level, and the cephalic index used to determine the braquicephaly level [5].

If any of these index are severe, the usage of a cranial orthosis is recommended. That orthosis tailor is based on each patients head shape and has to be used ideally 20 hours per day from 6 to 12 months usually. Every cranial orthosis is similar to a conventional helmet with two layers; a rigid one to keep the symmetry shape desired and a soft one which is in contact with the patient's skin and is in charge to make the pressure needed.

The first device destined to treat plagiocephaly was registered in the World Intellectual Property Organization (WIPO) on 1988 under the name of "Therapeutic and protective infant helmets” in US [6] [7]. Fig.2 shows the first registered helmet in WIPO.

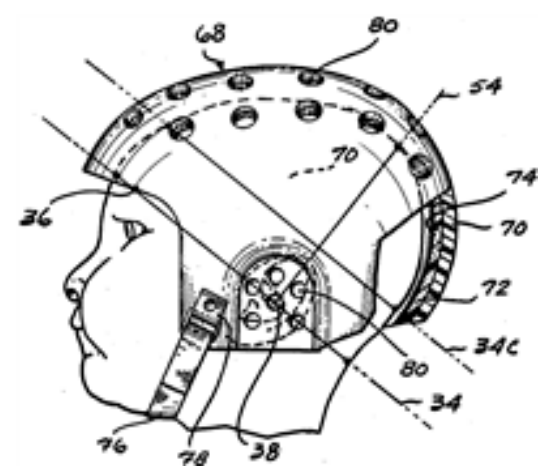

Figure 2: First Helmet registered at WIPO database

Moreover, there are other patent registrations. Since that year some of them also were focused on material ventilation and gripping ways, and devices to obtain molds from babies and its subsequent construction.

Regarding to sensorised helmets, there are some registrations on 2013 in the United States Patent and Trademark Office (USPTO). These ones incorporate pressure sensors [8], which are different from the sensor proposed in the present work and which main objective is to measure the time of use of the helmet.

When analysing the commercial distribution of the orthopaedic devices, the United States is the country that stands out more, nevertheless, in most of the European countries, the orthopaedic helmets can be bought with an average price of $2000 €$.

Finally, it could be interesting to mention some of the adherence systems to treatment that have been developed so far, which are generally focused on pharmacological treatments. The adherence methods are classified into different types as follows:

- Technical: by simplifying the doses to only one per day or simplified packs for daily doses, electronic tracking systems are alarms, with or without sensors, that count the amount of remaining medicine, and then, the alarms are triggered in case of 
unexpected behaviour.

- Behaviour: consist of phone reminders, mobile apps [9], mailing, home visits to patients, etc. Moreover, it has worked with reward based systems (gamification) on this type of methods.

- Educational: consist of offering information to patients in order to have a greater understanding of their disease, as information for diabetes or hypertension. The assistance to support groups is also found as treatment adherence methods [5].

- Multi-faceted: is a combination of two or more methods. In the majority of the reviewed articles, the researchers warn about the lack of any fixed protocol about adherence tools on both, adherence to pharmacological treatments and non-pharmacological [10] [11].

\section{Challenges and objectives of the sensorised helmet}

The key age to treat these type of deformities is between 4 and 18 months old, since after the skull is consolidated and it is not possible to perform corrections. See Fig. 3 for further information. Moreover, each helmet is tailored to the patient and it can be made of plastic or externally reinforced with glass fibre. The inside structure is covered with orthopaedic rubber. It must be used during almost all the day during 6 months in average. To provide tracking every 5 weeks the patient is cited to see the evolution and adjust the helmet, so the baby head continues with its natural growing [12].
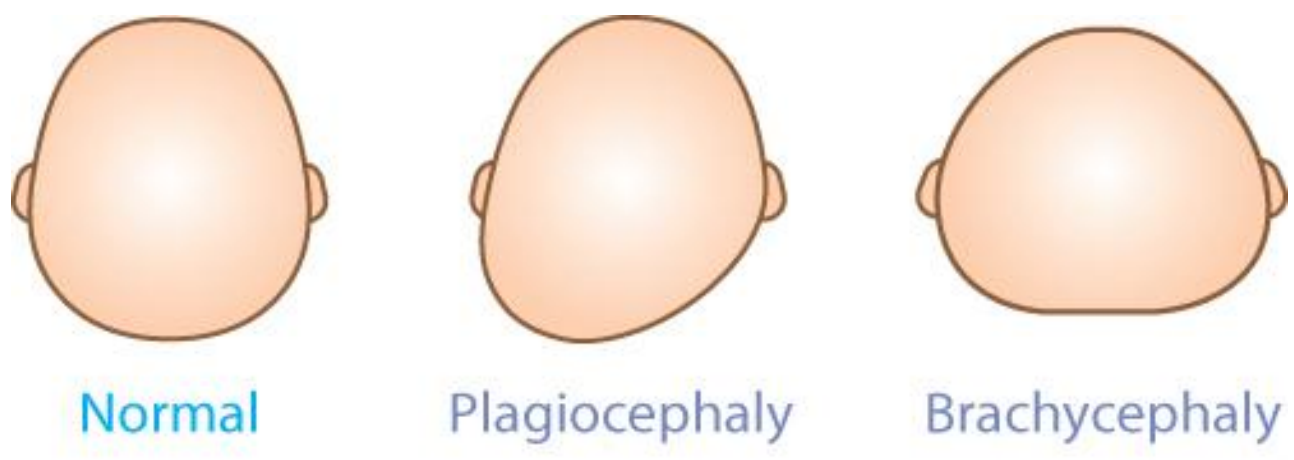

Figure 3. Cranial malformations

This system about the time of use and treatment adherence that has been designed, can be applied in many other medicine cases, as it can be for the use of splints or other orthopaedic devices and it will be very useful to verify certain results about medicine investigation protocols.

On the other hand, the use of fabric along the project evolution, different troubles to be solved have been found. Initially, what is being looked for is that all the babies have access to 
the treatment, so for the case of Mexico it is not possible to find centres where they can be assisted, in contrast with countries as Spain, US, United Kingdom or France to name some of them. This situation forces the parents to move on to United States, or in the worst case, let the baby suffer without being attended.

The FIX YOU engineers, by taking advantage of technology and engineering, have worked on a product that people living at economies as Mexico and Latin American countries can afford, but having, at the same time, the quality of the security that they are using and that will take care for their babies' health.

The next problem that was identified, already with multiple patients wearing the helmet, is the adherence to treatment, which is the key to accomplish a correction. Nowadays, it is not possible to know the amount of hours that the device has been used and we must trust that the information provided by the family about the adherence on the helmet of the baby is correct (either for a lack of memory or reality distortion). For this reason we are working on a usage sensor, located at strategic points of the helmet, which will be capable of defining if the device is well-placed or not and the time it is being used. To achieve this goal, different work tasks have been done collaboratively with a company called Alteraid, introducing the idea of the utilization timing counter, a mobile app implementation and data synchronization with the cloud. This scenario will allow to all stakeholders interested in the baby treatment to provide tracking of the usage of the helmet from any place.

In addition, conductive wires have gained popularity during the last years due to the rise of the wearable devices. Nevertheless, there are few projects that merge the use of conductive textiles with any adherence system or treatment registration.

Finally, it is intended as an objective, giving dissemination about the treatment and prevention of these type of deformities, so it has been found that from a global level it does not exist any protocol to attend these cases.

\section{System architecture}

A family with a baby suffering a cranial malformation, and joining the FIX YOU pilot tests, uses a sensorised orthopaedic helmet during multiple hours a day. Additionally, all the people involved in the welfare of this baby (family, medical staff, among others) can receive adherence information that comes from the helmet and which has been transmitted to a cloud that stores different health data of the baby. This platform, provided by Alteraid, allows to share this information with all the users interested in the baby's welfare.

The proposed system integrates pressure sensors inside the orthopaedic helmet. The collected information by these sensors is processed in a micro controller (based on arduino's technology) [13] which is mounted in the form of a small box to the orthopaedic device and then transmitted to a mobile device via Bluetooth (BluetoothLE) [14]. This mobile will be the responsible of sending the information to the cloud where it will be available to all the people interested in the patient's treatment; the patient itself when possible, relatives, caregivers and 


\section{Macrothink Institute ${ }^{\mathrm{TM}}$}

doctors, generating a network where all the people involved in the treatment of the patient can exchange information and social content from anywhere.

Given that, this system is expected to be used by the relatives and for this reason its use must be straightforward. Consequently, during each development stage, the technical complexity, the usability and the final user experience are taken into account.

In the platform (see figure 4) the social ecosystem (cloud) is the central part of the system and is composed as a SaaS (Software as a service) system. This is an important component because is mandatory to process al the acquired information from the input methods (sensors, mobile applications, etc). The acquired data is heterogeneous and modelled to fulfil the platform requirements like anonymity.

The main requirement needed in the social ecosystem is the user experience and to be transparent for the users, as a black box where they can do inputs and obtain processed information/data. To get the access to this processed data, this module provides an application interface (API) used for the data acquisition and data consumption as well.

The data is modelled and structured in a data graph, it grants versatility in the organization and helps user to reach the anonymity by the creation of "bonds" and share only the information which users wants to share with other.

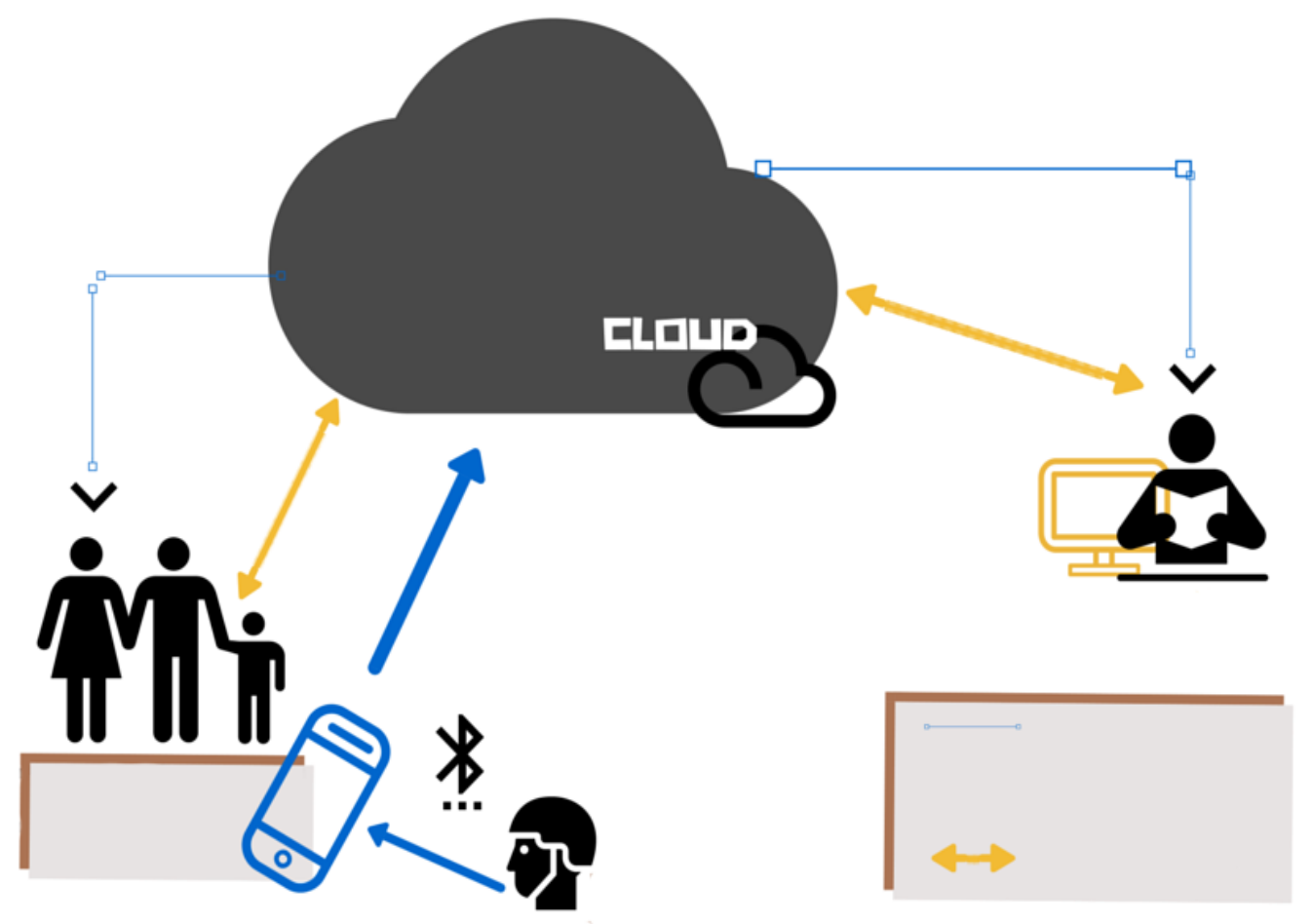

Figure 4. General system architecture

To understand it in more detail, the adherence registration system to the orthopaedic device must consider the following sections: 
- Sensors

- Follow up and treatment

- Analysis and dissemination of the information

\section{A. Sensors}

The proposed sensors for the system are pressure sensors based on fabrics and conductive wires (Ripstop). This type has been chosen because it will be directly in contact with the skin and its characteristic sweating. Also it may come into contact with water when cleaning the device. This measure will avoid its weathering and malfunction. The sensors have been located in strategic positions, as shown in Fig. 5, that besides providing information to count the utilization hours and adherence to treatment, also indicate if the device is well-placed. Both sensors are integrated inside the helmet and for the user are almost imperceptible.

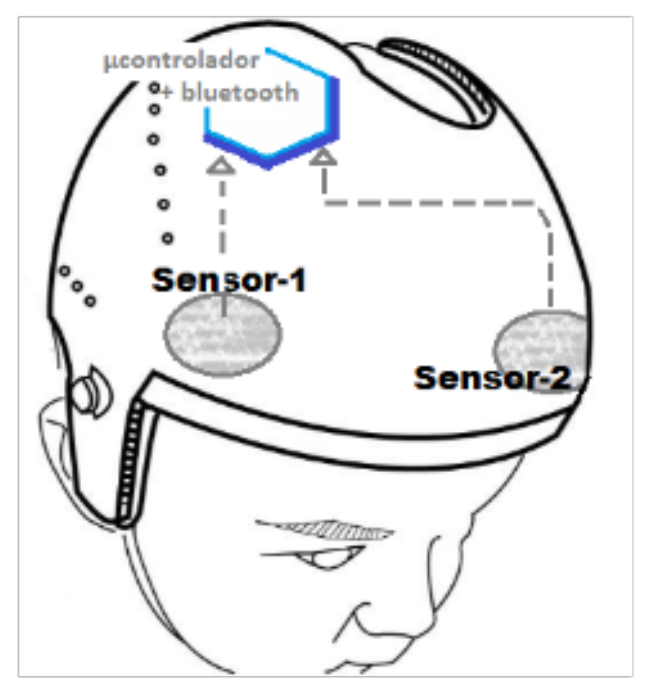

Figure 5. The helmet

\section{B. Follow up and information treatment}

Once the sensors are coupled, their signals go to a micro controller in digital mode. For this work an AVR has been chosen and for the preliminary tests the development platform “Arduino One" has been selected.

Initially, the micro controller is configured to the minimum energy consumption, waiting the activation through the interruption routine of any of the sensors. After the activation of the first sensor it asks about the second sensor with the objective of identifying if the orthopaedic device is well-placed. When the information of both is positive, the account time in minutes is initiated. While the sensors are active and the micro is counting the time, it turns on and off 


\section{Ml Macrothink}

intermittently, in order to optimize the chip power supply, until the signal of one of the both sensors is not received. The value of the elapsed time is stored in the register of a non-volatile memory.

To accomplish the transmission of the value of the time in use is necessary to be activated from a switch. The information transmission is done using a Bluetooth HC-05 module that is synchronized with the mobile app. The mobile application has been implemented for the android operating system and nowadays it is a proof of concept that allows to be linked to the Bluetooth device, receive the adherence information of the helmet (a time counter that represents the time while the helmet has been used) and the helmet identification. Once this information is received, and if there is connectivity available, this information is sent to the data repository (cloud). In the case the mobile phone does not have connectivity available, that information is stored in a local objects oriented database (db4o).

When there is connectivity, the mobile app sends all the information using secure HTTP (https) and an access token, that has been achieved previously through the OAUTH 2.0 negotiation, and that deploys as an authorization mechanism over the API in the data repository.

So sending the information to the cloud is authorized and it is prevented the users to distort the adherence information of a helmet, since all the process is transparent for them. On the other hand, the families of the babies with malformations are involved deeply in the wellness of their baby and it is not the type of user who distorts information, rather they need support tools to manage the information and avoid human memory errors. Fig. 6 shows the flux diagram of the operation described before.

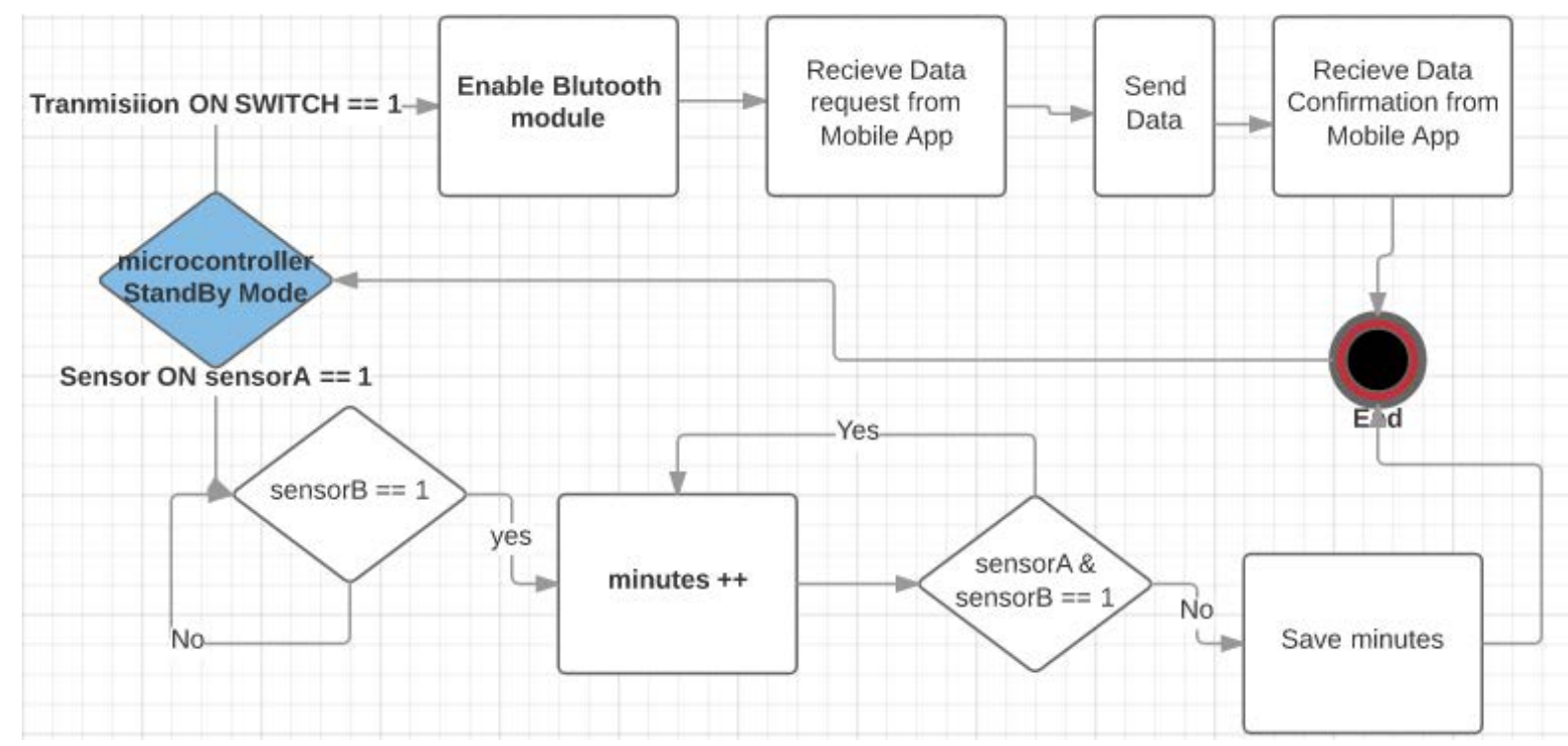

Figure 6. Flux diagram

Nowadays, the project is in a pilot phase and the micro controller (based on arduino's technology) and the sensors can be supplied by AA batteries (that can be substituted), or a 
LiPo battery, which can be charged if necessary.

The micro controller can monitor the battery state and perform alerts through a LED light and/or notifications to the mobile app. So it can be assured that the adherence system to the helmet can operate during its treatment (6 months approximately). On the other hand, the adherence information of the helmet is stored in a non-volatile memory of the micro controller, so if the battery is depleted the information is not lost.

\section{Analysis and information broadcast}

In the architecture, the cloud is the central part of the system and it is defined as SaaS (Software as a Service). This is an important component because it allows processing the required information through different input methods (sensors, mobile apps, etc).

In order to achieve the access to these processed data, the module provides an application interface (API) based on REST, which is used to data acquisition and its consumption. This platform has been designed to offer access to third party, that is to say, the applications, the services or the sensors through an Application Programming Interface (API) for the output and input of the information. To ensure the access authorization mechanisms to the API, an authorization mechanism based on OAuth 2.0 is used.

\section{Data Model}

The information of the social ecosystem stored in the cloud can be shown to the interested users through a browser or with a mobile app. In Fig 7, it can be seen that a user, for example the father, can access health information from the members of the family, such as his son. Typically, three types of different actors are identified, although they may exist others in the future:

- Family members: they are users linked to a baby with a cranial malformation. The baby will not be a user in this system although in a future, when he grow up, he could be a user and also visualize all the information related with himself.

- Medical staff: the medical staff could desire to have access to the patient's' information and in that way having a good tracking of their treatments. At the same time the medical staff can obtain aggregated information about the patients.

- Other community members: people with some relation with the baby. For example caregivers. 


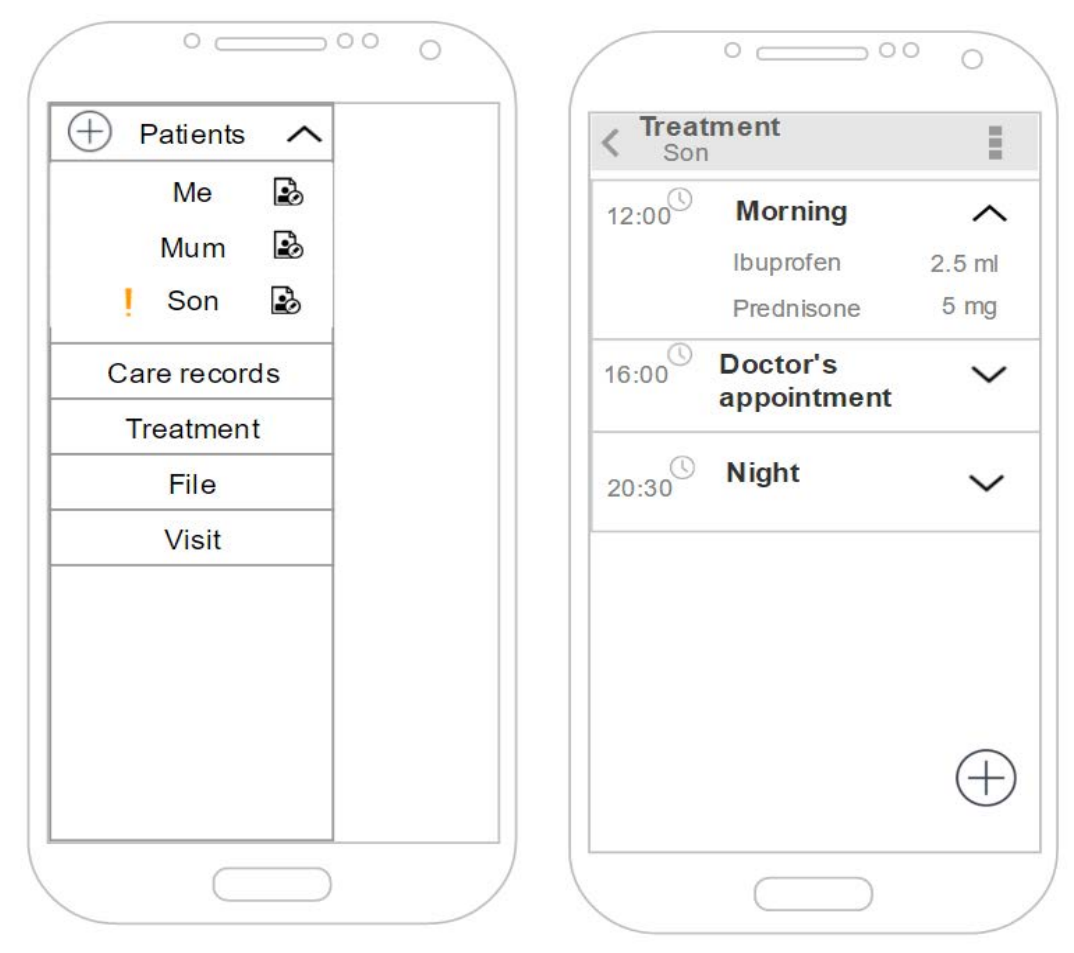

Figure 7. Application with son’s information

The information is modelled and defined in a data structure based on graphs. The nodes of this graph represent a type of information that is related between itself according to a hierarchy criteria and sharing relation. Each node stores information in a schema-less paradigm that allows the existence of different sets of fields for each node. This type of information organization allows being flexible and adapting to the new requirements. This scheme provides versatility in the organization and helps to organize the information in an efficient way.

The main information entities of the data model are the following:

- Users: it is one of the main components of the platform that manages the relevant information of a physical person as the name, contact data (phones, email) to the notifications and credentials for accessing the platform.

- Bond: it is a virtual element that allows storing information or a part of it that will be stored and shared with other users.

- Measures: is the element where the values are stored. The measure configuration is specified during its creation. The type of data can be determined (numeric, character array, etc) and meta-information as range of values to launch notifications, alerts or big data.

The following picture (figure 8) shows an example of the data model and the relationship between the different compounds of it, using the model defined previously. 


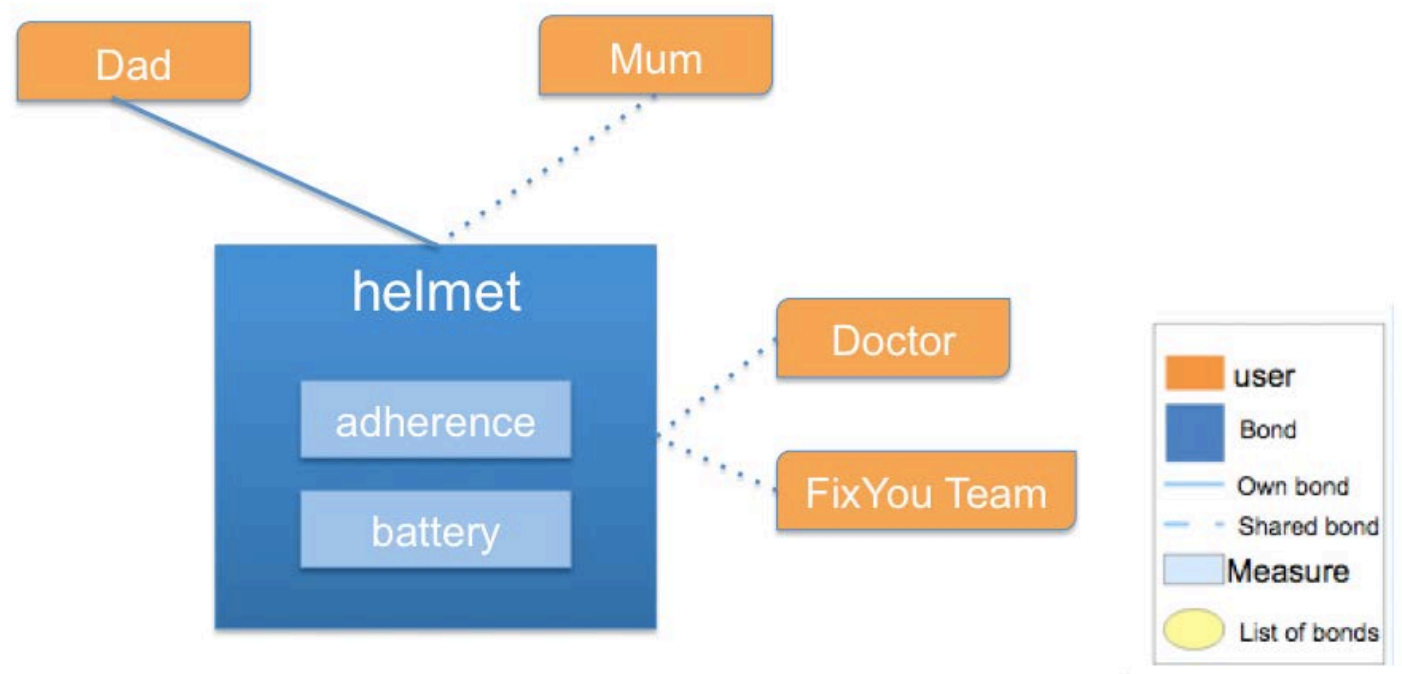

Figure 8. Data model

As it has been described before, the nature of the information is hierarchical, and for this reason and after an analysis of the different database options, the information has been determined to be stored in a NOSQL (Not-only) [15] database based on graphs (Neo4j) that is specially designed to store models based on graphs and big volume of information.

In this project, the information organization has been defined as follows. The baby (and his information associated to the helmet) will be a bond whose owner will be a user (for example, a parent). The bond owner will be the person who decides the sharing policies of the information, or part of it, stored in this virtual element. For example, this bond, which represents and stores information of a helmet of a determined baby, can be related with different users: relatives, the two neurosurgeons paediatricians, pediatric nurses and the babysitter.

For the data structure purposes, a node of type user will exist (the owner or the supervisor of the information) that will be related with nodes of type bond and one of them will be his baby. This bond will include a set of measures and one of them can manage the adherence to the helmet use. Additionally, other measures can be stored, as the measure of the cranial size, the baby weight, etc. These information can be shared with other users and this will imply the graph to be enriched with new relations between the recent users and the links.

\section{Results}

Currently, the device has been tested with 60 patients diagnosed with plagiocephaly, without the adherence module incorporated. In the future study, different proofs of the adherence system will be performed with new patients (communications module and mobile app) and the effectiveness of the treatment in both groups may be evaluated.

Next, in Fig. 9 and Fig. 10, the results of two patients are presented, Px1 and Px2. The 


\section{Macrothink}

treatment was performed with the accompaniment of a pediatric neurosurgeon doctor. For the Px1, who is a female patient diagnosed with plagiocephaly, the treatment started with 10 years old. The data was collected every 30 days adding a total of 11 meetings. On the other hand, Px2, who is a male patient diagnosed at 6 months old with brachycephaly, 7 tracking meetings are presented.

The following tables show the evolution of the oblique cranial index computed. This index indicates the head symmetry grade of the patient (Px1). According to medical literature, an index of $104 \%$ or lesser corresponds to a normal or symmetric head.

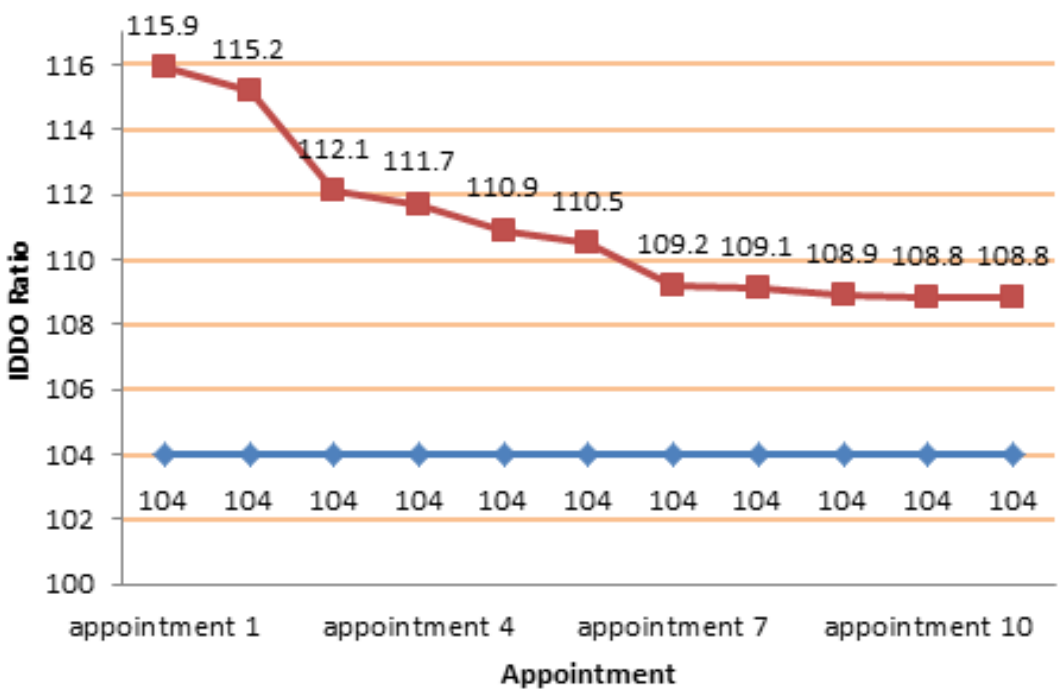

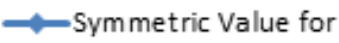

Plagioce phaly

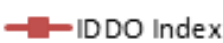

Figure 9. Results of patient 1

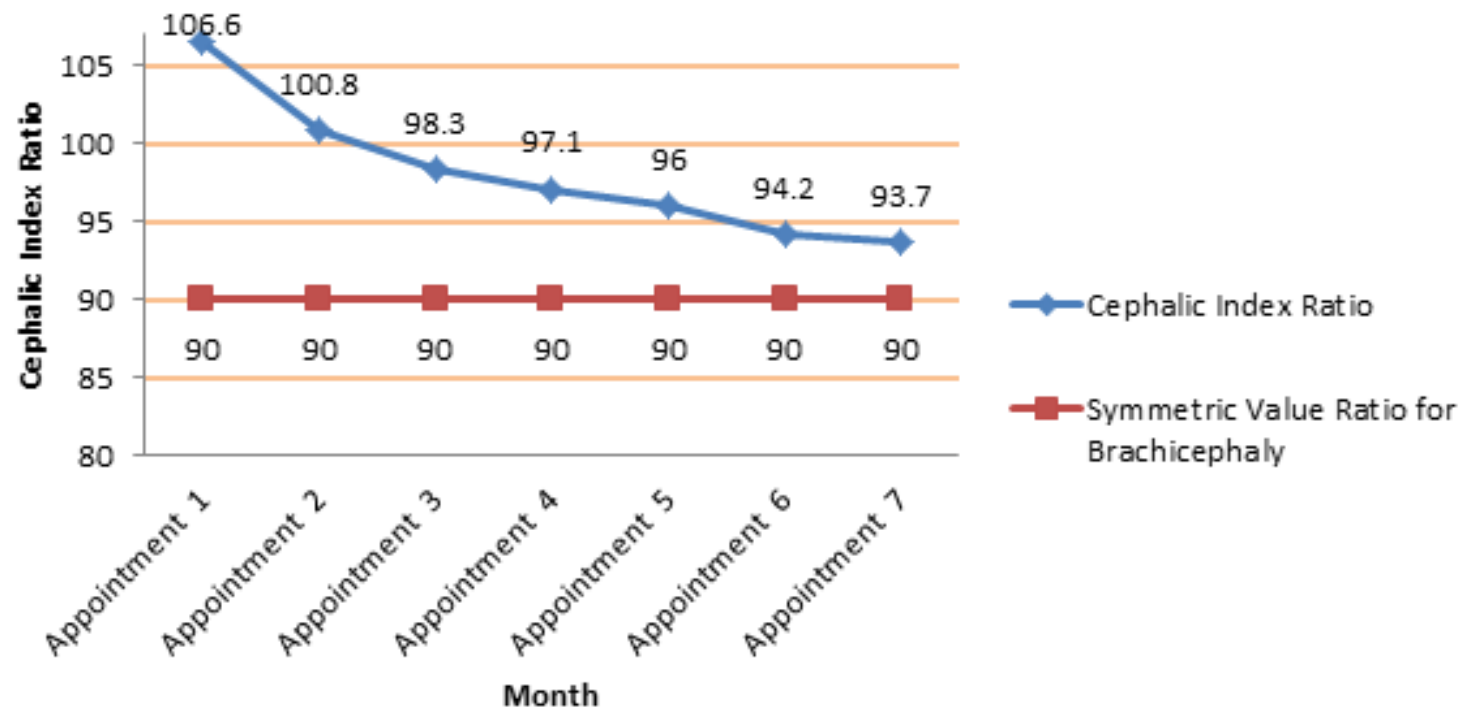

Figure 10. Results of patient 2

The malformation or asymmetry of both patients tends to decrease approaching to the 
symmetric value for each type of deformation.

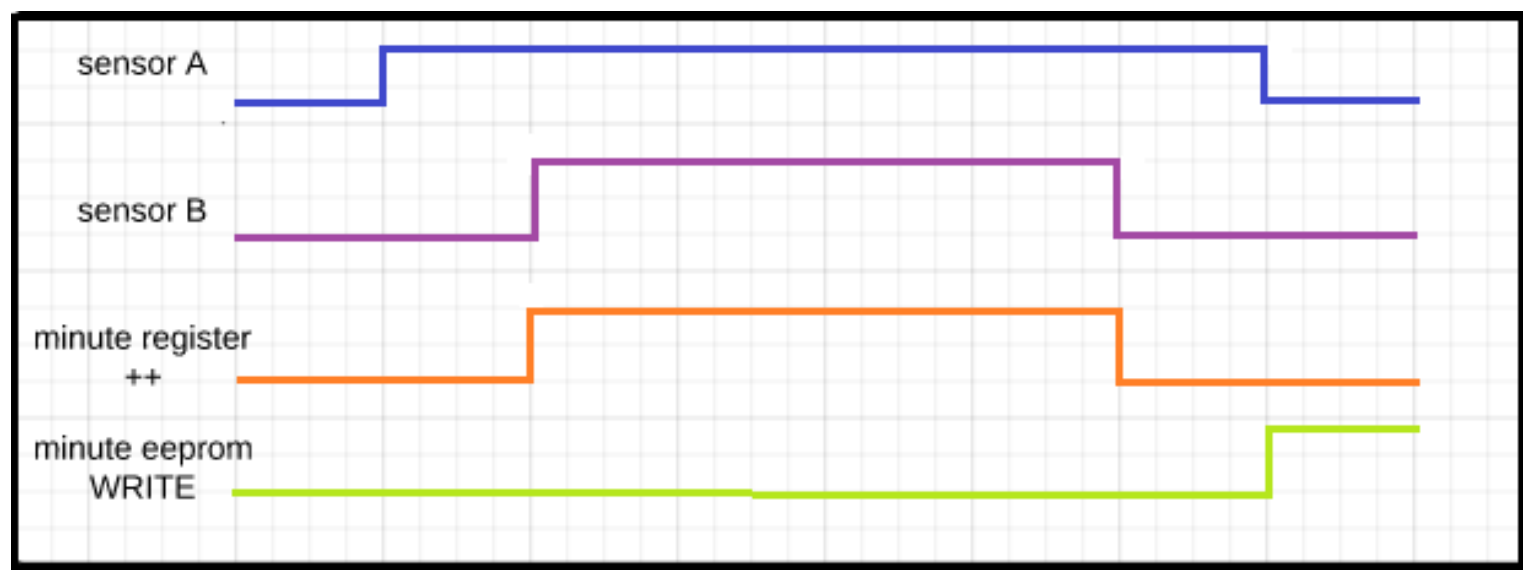

Figure 11. Boolean system time diagram

The diagram above shows how work the integrated stages of the system developed in the present paper; sensors and micro controller “Arduino One” algorithm.

\section{A. Expected results}

As the time data are available, the hypothesis of the larger the time of use, the faster will be the correction, can be confirmed. This will be reflected in the slope value of the straight corresponding to the cranial value.

\section{Conclusion}

The papers shows how integrating frequently used algorithms such as a sensor sending a digital O/I, time interval counting, data Bluetooth transmission, or sharing data in the cloud can turn into a complex system capable of monitor 24/7 a treatment string along with the patient.

As indicated before, until today 60 patients between 4 and 18 months old have been treated. Moreover, they have used the helmet and the majority have achieved successful results, understanding as successful that the shape of their head have recovered the symmetry. Fig. 12 shows the actual usage examples. Incorporating the adherence module would encourage parents to be more constant; reducing the time of usage in terms of months that will result less uncomfortable for babies. On the other hand this Adherence Module will provide useful data for physicians and investigators also researching about plagiocephaly treatment that currently is not found in any articles, further results might origin statistical models that could predict treatments duration and results. 


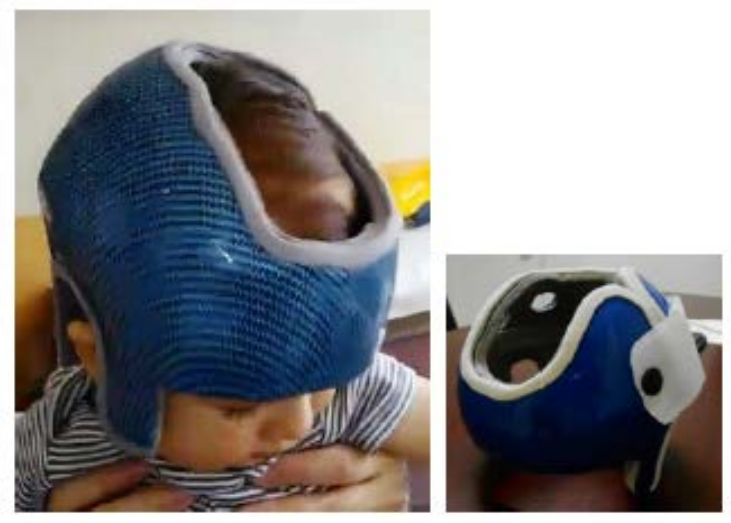

Figure 12. Real index

Further work is needed to have a functional accurate sized prototype to test on at least 5 patients and present and provide results, and identify algorithms to be improved. The advantage of this proposed model is that the sensors are imperceptible for the patient, and these data acquisition does not depend on the user. It is done automatically. For the case of the data transmission from the orthopaedic device to the mobile phone, the person must be involved (the baby could not do that). On the other hand, the micro controller has a backup memory, and the reminders are sent through a mobile app for the synchronization. This adherence module supported in the cloud is not only applied in this specific helmet device, but in addition other orthotic devices will be able to identify causes that lead patients to not accomplish the adherence desired in treatments as well as to improve their health and wellness.

\section{Acknowledgement}

FIX YOU has got the opportunity of working with several institutions and organizations. FUMEC México-USA; Idea Foundry in Carnegie Melon University, Pennsylvania-USA, Televisa Foundation, México. And as it has been said, FIX YOU is currently working with Alteraid, thanks to the program RedEmprendia Booster-e for new entrepreneurs.

Additionally, FIX YOU has been recognized in the following competitions:

- Iberoamerican Award for Innovation and Entrepreneurship by Secretary General Iberoamerica (SEGIB), Spain 2013.

- Higher Education Foundation Company (FESE), Mexico 2014.

- Enterprise innovation award - Bank of Santander SA, Mexico 2014.

\section{References}




\section{Macrothink}

Network Protocols and Algorithms

ISSN 1943-3581

2016, Vol. 8, No. 1

[1] Trip Database Limited, What is the role of helmet therapy in positional plagiocephaly? , TRIP Database Limited, 2009.

[2] DeGrazia, M; Giambanco, D; Hamn, G; Ditzel, A; Tucker, L; Gauvreau, K Prevention of Deformational Plagiocephaly in Hospitalized Infants Using a New Orthotic Device Jognn-Journal of Obstetric Gynecologic and Neonatal Nursing. Volume: 44 Issue: 1 Pages: 28-41 DOI: 10.1111/1552- 6909.12523. Published: JAN-FEB 2015

[3] Kim,SY; Park, MS; Yang, JI; Yim,Comparison of Helmet Therapy and Counter Positioning for Deformational Plagiocephaly. SY. Annals of Rehabilitation Medicine. Volume: 37 Issue: 6 Pages: 785-795 . Published: 2013

[4] Lee, A; Van Pelt, AE; Kane, AA; Pilgram, TK; Govier, DP Comparison of perceptions and treatment practices between neurosurgeons and plastic surgeons for infants with deformational plagiocephaly Neurosurg Pediatr. 2010 Apr;5(4):368-74. doi: 10.3171/2009.11.PEDS0983.

[5] Rogers G.F., Miller J., Mulliken J.B., Comparison of a modifiable cranial cup versus repositioning and cervical stretching for the early correction of deformational posterior plagiocephaly, Plastic \& Reconstructive Surgery: March 2008 - Volume 121 - Issue 3 pp 941-947. doi: DOI: 10.1097/01.prs.0000299938.00229.3e. March 2008.

[6] Villani D., Meraviglia M.V., (2014) pp12-14, Positional Plagiocephaly italy: Springer.

[7] Sterling, C. (1988) First Helmet registered at Wipo database [figure 2] patentscope.com.int.

[8] G.P. Bernardoni; “Cranial Helmet for Infants”, U.S. patent 2013/0289459, October 31, 2013

[9] L. Dayer, S. Heldenbrand, P. Anderson, P. O. Gubbins, B. C. Martin Medication Adherence Apps. Potential Benefits to Patients and Providers Journal of the American Pharmacists Association, 2013;53(2):172-181.

[10]S; Sluijs, E; Van Dijk,L; De Ridder, D; Heerdink, R; Bensing, J Patient adherence to medical treatment: a review of reviews Van Dulmen BMC Health Services Research 2007, 7:55

[11]Hall, AM; Kamper, SJ; Hernon, M; Hughes, K; Kelly, G; Lonsdale, C; Hurley, DA; Ostelo, R Measurement Tools for Adherence to Non-Pharmacologic Self-Management Treatment for Chronic Musculoskeletal Conditions: A Systematic Review Archives of Physical Medicine and Rehabilitation. Volume: 96. Issue: 3. Pages: 552-562. DOI: 10.1016/j.apmr.2014.07.405. March 2015

[12]Head Shapes www.flatheadsyndrome.org.uk/media/1024/head-shapes.jpg [last access 24/03/2016]

[13]Arduino https://www.arduino.cc/ [last access 21/03/2016]

[14]Bluetooth https://www.bluetooth.com/what-is-bluetooth-technology [last access 
[15]No-SQL database http://nosql-database.org/ [last access 21/03/2016]

\section{Copyright Disclaimer}

Copyright reserved by the author(s).

This article is an open-access article distributed under the terms and conditions of the Creative Commons Attribution license (http://creativecommons.org/licenses/by/3.0/). 